\title{
Photon-assisted mesoscopic transport through a toroidal carbon nanotube coupled to
}

\section{normal metal leads}

\author{
Hong-Kang Zhao ${ }^{1,2}$, Jian Wang ${ }^{1}$ \\ 1. Department of Physics, The University of Hong Kong, Pokfulam Road, \\ Hong Kong, China \\ 2. Department of Physics, Beijing Institute of Technology, \\ Beijing 100081, China *
}

(March 4, 2008)

The mesoscopic transport through a toroidal carbon nanotube (TCN) system applied with ac fields to the electrodes has been investigated by employing the nonequilibrium Green's function (NGF) technique. The Landauer-Büttiker -like formula is presented for numerical calculations of differential conductance and tunneling current. The conductance resonance takes place due to the electrons resonating in the quantum levels of TCN and side-band caused by the external ac fields. The photon-assisted transport can be observed both in conductance oscillation and current oscillation with respect to the magnetic flux. The side peaks and current suppressions are the main effect of photon absorption and emission in the transport procedure. The stair-like current-voltage characteristics are resulted from the quantum nature of TCN and applied microwave fields. The photon-electron pumping effect can be obtained by applying the microwave fields to the leads.

PACS: 73.40.-c; 73.63.Fg; 73.61.Wp; 73.22.-f

The electronic properties of carbon nanotubes $(\mathrm{CN})$ attract much attention of physicists and electronic engineers due to their profound physical connotations and potential applications. The metallic and semiconducting behaviors are intimately related to the diameter and chirality of a $\mathrm{CN}[1-4]$, and the metal-semiconductor transition takes place by applying an external magnetic field $[5,6]$. The material and shape of devices play central roles for the feature and quality. Recently, the development of nano-technology provides the possibility for fabricating various nano-devices. $\mathrm{CNs}$ are prospective materials of future electronic devices due to the metallic and semiconducting behaviors. Some electronic devices like field effect transistors [7,8] and diodes [9] have been fabricated for the application of point view. This opens up a new artifical laboratory to study low dimensional transport properties, and provides novel physical prediction for the further application [10-16]. The toroidal carbon nanotube (TCN) is a form of carbon structure, which is a torus structure by bending the carbon tube such that the two edges are connected. The tori proposed are constructed by introducing a single pentagon-heptagon pair into the perfect hexagon bonding pattern to connect car- 
bon tubules, and its construction can be based on the $C_{60}$. The theoretical [17-19] and experimental [20] investigations on the electron properties of carbon toroid revealed the quantum nature of quasi-one-dimensional ring. Compared with normal metal or semiconductor ring, the TCN can carry larger persistent current due to the modification of energy structure and energy gap [21], and it provides rich physical properties due to its structure. The TCN coupled with normal metals (N-TCN-N), the Andreev reflection in the TCN coupled to normal metal and superconductor (N-TCN-S), and the dc Josephson current through the system with a TCN coupled to two superconducting leads (S-TCN-S) [22] have been investigated to display novel electronic transporting properties. TCN is threaded with a static magnetic flux $\phi$ which induces Aharonov-Bohm-like effects. The conductance and tunneling current are evaluated to show the resonant and oscillating behaviors associated with magnetic flux. The Aharonov-Bohm magnetic flux controls the tunneling current since it causes the metal-semiconductor transition in the TCN. In this paper, we investigate the photon-assisted mesoscopic transport through the TCN coupled to two normal metal electrodes (N-TCN-N) system by imposing microwave fields to the leads. Since the system is perturbed by the microwave fields, the tunneling current is characterized with the oscillating features of the external microwave fields. The external microwave fields split the channels to form side-bands, and electrons resonate with the photon energies. We employ the Green's function (NGF) technique to calculate the tunneling current[23-25].

The TCN is formed by rolling a finite graphite sheet from the origin to the vectors $\mathbf{R}_{\mathrm{x}}=\mathrm{m}_{1} \mathbf{a}_{1}+\mathrm{m}_{2} \mathbf{a}_{2}$, and $\mathbf{R}_{\mathbf{y}}=\mathrm{p}_{1} \mathbf{a}_{1}+\mathrm{p}_{2} \mathbf{a}_{2}$ simultaneously, where $\mathbf{a}_{1}$ and $\mathbf{a}_{2}$ are the two primitive lattice vectors possessing the same magnitude as $a=\left|\mathbf{a}_{1}\right|=\left|\mathbf{a}_{2}\right|=\mathrm{b} \times 3^{1 / 2}$. Here $b=1.44$ $\stackrel{\circ}{A}$ is the $\mathrm{C}-\mathrm{C}$ bond length of CNs known to be slightly larger than that of graphite [3]. We denote the TCNs by $\left(m_{1}, m_{2} ; p_{1}, p_{2}\right)$ as convention. The TCN satisfies the periodical boundary conditions along both of the longitudinal and transverse directions. Two kinds of TCN with highly symmetric structures are armchair $(m, m ;-p, p)$ TCN and zigzag $(m, 0 ;-p, 2 p)$ TCN. The armchair TCN possesses the symmetry with armchair structure along the transverse direction and zigzag structure along the longitudinal direction. The zigzag TCN has the structure in both of the directions being zigzag. We denote the diameter of $\mathrm{CN}$ as $d_{t}$, and the diameter of mesoscopic ring as $D_{t}$. The diameters of the armchair $(m, m ;-p, p)$ TCN are $d_{t}=3 b m / \pi$, and $D_{t}=3^{1 / 2} b p / \pi$; the diameters of the zigzag $(m, 0 ;-p, 2 p)$ are given by $d_{t}=3^{1 / 3} \mathrm{bm} / \pi$, and $D_{t}=3 b p / \pi$. In the absence of magnetic flux, the armchair TCN is a metal as $p=3 \nu$ (type I TCN), while it is a semiconductor with narrow energy gap as $p=3 \nu \pm 1$ (type II TCN) where $\nu$ is an integer. The zigzag TCN exhibits semiconductor behavior with large energy gap in 
the order $E_{g}=0.1 \sim 1.0 \mathrm{eV}$. We investigate the system with the diameter ratio of the nanotube $d_{t}$ to the diameter $D_{t}$ of mesoscopic ring being much smaller than 1 , i.e., $\kappa=d_{t} / D_{t}<<1$.

The system is composed of three parts: the central TCN, and the normal metal leads. The central TCN is applied with a static magnetic field $\mathbf{B}$ perpendicular to the ring, which induces a magnetic flux $\phi$ threaded through the TCN. The electrons are free from the magnetic field $\mathbf{B}$, but the vector potential $\mathbf{A}$ affects the behaviors of electrons due to the Aharonov-Bohm effect. The electrons of the leads are described by the grand canonical ensembles, and the central TCN is described by the tight-binding Hamiltonian. We consider the circumstance that the two leads are biased by the dc voltage $V$ which is the drop of chemical potentials of the two leads $\mu_{L}-\mu_{R}=e V$. The microwave field with frequency $\omega_{\gamma}$ is applied to the $\gamma$ th lead forming potential drop $e \tilde{V}_{\gamma d} \cos \left(\omega_{\gamma} t\right)$ in it. So that the electron energy in the $\gamma$ th lead is described by the time-dependent one $\epsilon_{\gamma k}(t)=\epsilon_{\gamma k}+e \tilde{V}_{\gamma d} \cos (\omega t)$ in the adiabatic approximation. In the diagonalized representation of TCN, the electronic properties can be determined by the total Hamiltonian of the system, which is the summation of the three sub-Hamiltonians and the tunneling interaction term

$$
\begin{array}{r}
H=\sum_{\gamma k \sigma} \epsilon_{\gamma k}(t) a_{\gamma, k \sigma}^{\dagger} a_{\gamma, k \sigma}+\sum_{j \ell \delta \sigma} E_{\delta, \ell j}(\phi) c_{\delta \sigma, j \ell}^{\dagger} c_{\delta \sigma, j \ell} \\
+\sum_{\gamma k \sigma} \sum_{j \ell \delta}\left[R_{\gamma \delta, j \ell}^{*}(k) c_{\delta \sigma, j \ell}^{\dagger} a_{\gamma, k \sigma}+H . c .\right]
\end{array}
$$

where $a_{\gamma, k \sigma}^{\dagger}\left(a_{\gamma, k \sigma}\right)$, and $c_{\delta \sigma, j \ell}^{\dagger}\left(c_{\delta \sigma, j \ell}\right)$ are the creation (annihilation) operators of electron in the two leads and TCN, respectively $\cdot R_{\gamma \delta, j \ell}(k)$ is interaction strength of particles between the $\gamma$ th lead and TCN. $E_{\delta, \ell j}(\phi)$ is the energy of TCN, which is intimately associated with the structure of concrete TCN. The energy of armchair TCN in tight-binding approximation is given by [21]

$$
\begin{array}{r}
E_{\delta, \ell j}(\phi)=\delta \gamma_{0}\left\{1+4 \cos \left(\frac{\pi j}{m}\right) \cos \left[\frac{\pi\left(\ell+\phi / \phi_{0}\right)}{p}\right]\right. \\
\left.+4 \cos ^{2}\left[\frac{\pi\left(\ell+\phi / \phi_{0}\right)}{p}\right]\right\}^{1 / 2}
\end{array}
$$

The energy of the zigzag TCN in the tight-binding approximation is given by

$$
\begin{array}{r}
\tilde{E}_{\delta, \ell j}(\phi)=\delta \gamma_{0}\left\{1+4 \cos \left(\frac{\pi j}{m}\right) \cos \left[\frac{\pi\left(\ell+\phi / \phi_{0}\right)}{p}\right]\right. \\
\left.+4 \cos ^{2}\left(\frac{\pi j}{m}\right)\right\}^{1 / 2}
\end{array}
$$

where $j=1,2, \ldots, m ; \ell=1,2, \ldots, 2 p ; \delta= \pm, \gamma_{0}=3.033$ $\mathrm{eV}$, and $\phi_{0}=h / e$ is the flux quantum. We employ the NGF technique to evaluate the current formulas. The detailed definitions of Green's functions and derivation of the photon-assisted tunneling current can be found in Refs.[23-25], in which we have made gauge transform to remove the time dependent energy in the interaction strengths.

The time-averaged tunneling current can be expressed by the Fourier transformed Green's functions $G_{\delta \ell j \sigma}^{X}(\epsilon)$ as

$$
I_{\gamma}=-\frac{2 e}{h} I m \sum_{\delta \ell j \sigma} \int d \epsilon \Gamma_{\gamma}\left[f_{\gamma}(\epsilon) G_{\delta \ell j \sigma}^{r}(\epsilon)+\frac{1}{2} G_{\delta \ell j \sigma}^{<}(\epsilon)\right],
$$


where $f_{\gamma}(\epsilon)$ is the Fermi distribution function given by $f_{\gamma}(\epsilon)=1 /\left\{\exp \left[\left(\epsilon-\mu_{\gamma}\right) / k_{B} T\right]+1\right\}$ at temperature $T$. We define the retarded Green's function of the isolated TCN as $\mathbf{g}_{\delta \ell j \sigma}^{r}(\epsilon)=1 /\left[\epsilon-E_{\delta, \ell j}(\phi)+i \eta\right]$, where $\eta \rightarrow 0$. The retarded Green's function of TCN in the coupled system can be solved by employing Dyson equation $G^{r}=g^{r}+g^{r} \Sigma^{r} G^{r}$, where $\Sigma^{r}=\sum_{\gamma} \Sigma_{\gamma}^{r}$ is the selfenergy of the system defined by summing up the selfenergies in the two leads. The Keldysh Green's function of the coupled TCN is derived from the equation $G^{<}=g^{r}\left[\Sigma^{r} G^{<}+\Sigma^{<} G^{a}\right]$. The retarded, advanced and Keldysh self-energies of the system is related to the corresponding Green's functions by the relation $\Sigma^{X}(\epsilon)=$ $\sum_{\gamma m k} J_{m}^{2}\left(\Lambda_{\gamma}\right) g_{\gamma, k \sigma}^{X}\left(\epsilon-m \hbar \omega_{\gamma}\right),(X \in\{r, a,<\})$, where $\Lambda_{\gamma}=e \tilde{V}_{\gamma d} / \hbar \omega$. We assume that the electrons are equally coupled between different tunneling channels,ie., $R_{\gamma \delta, j \ell}(k)=R_{\gamma}(k)$, and the line-width of lead is defined by $\Gamma_{\gamma}(\epsilon)=2 \pi \sum_{k}\left|R_{\gamma}(k)\right|^{2} \delta\left(\epsilon-\epsilon_{\gamma k}\right)$. The Green's function of the coupled TCN system is therefore given by

$$
G_{\delta \ell j \sigma}^{r}(\epsilon)=\frac{1}{\epsilon-E_{\delta, \ell j}(\phi)-\Sigma^{r}(\epsilon)} .
$$

The density of state (DOS) of the $\gamma$ th lead is $\rho_{\gamma}(\epsilon)=$ $\sum_{k} \delta\left(\epsilon-\epsilon_{\gamma k}\right)$, and the retarded self-energy of this lead can be described by the imaginary and real parts as $\Sigma_{\gamma}^{r}(\epsilon)=$ $\sum_{m} J_{m}^{2}\left(\Lambda_{\gamma}\right)\left[\beta_{\gamma m}(\epsilon)-i \Gamma_{\gamma}\left(\epsilon-m \hbar \omega_{\gamma}\right) / 2\right]$, where

$$
\beta_{\gamma m}(\epsilon)=\left|R_{\gamma}\right|^{2} \int d \epsilon_{1} \frac{\rho_{\gamma}\left(\epsilon_{1}-m \hbar \omega_{\gamma}\right)}{\epsilon-\epsilon_{1}} .
$$

Similarly, we can obtain the Keldysh Green's function by solving the Dyson-like equation self-consistently to give the pseudo-equilibrium expression. Substituting the Green's functions above into the current formula (4), finally we obtain the current formula of the $\gamma$ th lead as

$$
\begin{array}{r}
I_{\gamma}=\frac{e}{h} \sum_{m n \sigma} \sum_{\beta \neq \gamma} \int d \epsilon T_{\gamma \beta \sigma, m n}(\epsilon)\left[f_{\gamma}\left(\epsilon-m \hbar \omega_{\gamma}\right)\right. \\
\left.-f_{\beta}\left(\epsilon-n \hbar \omega_{\beta}\right)\right],
\end{array}
$$

where the transmission coefficient of electron tunneling from the $\gamma$ th lead to the $\beta$ th lead is defined by

$$
\begin{array}{r}
T_{\gamma \beta \sigma, m n}(\epsilon)=\Gamma_{\gamma}\left(\epsilon-m \hbar \omega_{\gamma}\right) \Gamma_{\beta}\left(\epsilon-n \hbar \omega_{\beta}\right) \\
\times J_{m}^{2}\left(\Lambda_{\gamma}\right) J_{n}^{2}\left(\Lambda_{\beta}\right) \sum_{\delta \ell j}\left|G_{\delta \ell j \sigma}^{r}(\epsilon)\right|^{2}
\end{array}
$$

The transmission coefficient $T_{\gamma \beta \sigma, m n}(\epsilon)$ of the system denotes the electrons transporting from the $\gamma$ th lead in the $\mathrm{m}$ channel to the $\beta$ th lead in the $\mathrm{n}$ channel. The current conservation $\sum_{\gamma} I_{\gamma}=0$ is satisfied for the time-averaged current. The external microwave fields induce two effects: one is the splitting of electron level to form multichannels in the leads, and the other is to modify the chemical potentials of the leads. This mesoscopic transport contains the photon-electron pumping effect and the Aharonov-Bohm effect. The magnetic flux controls the energy gap $E_{g}(\phi)$ to form periodic oscillation of current with respect to $\phi$ in period $\phi_{0}$. By varying the magnetic flux $\phi$, the metal-semiconductor transition takes place, and the energy gap alternates with $\phi$.

At zero temperature, the tunneling current formula Eq.(6) is reduced to 


$$
I=\frac{e}{h} \sum_{\sigma} \sum_{m n} \int_{n \hbar \omega_{R}}^{e V+m \hbar \omega_{L}} T_{L R \sigma, m n}(\epsilon) d \epsilon .
$$

We have taken the chemical potential of the right lead $\mu_{R}$ as the energy measurement point by setting $\mu_{R}=0$. This means that the Fermi level of the TCN is zero as $e V=0$, and it is symmetric about this point. In the absence of microwave field, the electrons can not transport through the system as $V=0$ at zero temperature for the semiconducting TCN, since the energy gap $E_{g}(\phi)>0$ acts as the threshold to restrict the tunneling of electrons. However, in the presence of microwave fields, the tunneling current exhists as $\left|e V+m \hbar \omega_{L}-n \hbar \omega_{R}\right|>E_{g}(\phi) / 2$ $(n, m=0, \pm 1, \pm 2, \ldots)$. Therefore, the tunneling current is the result of compound effects related to the photonelectron pumping, Aharonov-Bohm effect, and the detailed TCN structure. For the leads broaden immediately at the connection to TCN, the line-width can be treated by wide-band limit, which means that the line-width is independent on the energy variable $\epsilon$, i.e., $\Gamma_{\gamma}(\epsilon)=\Gamma_{\gamma}$. In the wide-band limit, the retarded self-energy of the system $\Sigma^{r}=-i \sum_{\gamma} \Gamma_{\gamma} / 2$. For the symmetric situation $\Gamma_{L}=\Gamma_{R}=\Gamma$ and wide-band limit, the tunneling current at zero temperature is given by

$$
\begin{array}{r}
I=\frac{2 e}{h} \Gamma \sum_{m n} \sum_{\delta j \ell} J_{m}^{2}\left(\Lambda_{L}\right) J_{n}^{2}\left(\Lambda_{R}\right) \\
\times\left\{\tan ^{-1}\left[\frac{e V+m \hbar \omega_{L}-E_{\delta, \ell j}(\phi)}{\Gamma}\right]\right. \\
\left.-\tan ^{-1}\left[\frac{n \hbar \omega_{R}-E_{\delta, \ell j}(\phi)}{\Gamma}\right]\right\} .
\end{array}
$$

The differential conductance at zero temperature is determined by the Breit-Wigner resonant form

$$
\frac{d I}{d V}=\frac{2 e^{2}}{h} \sum_{m} \sum_{\delta j \ell} \frac{J_{m}^{2}\left(\Lambda_{L}\right) \Gamma^{2}}{\left(e V+m \hbar \omega_{L}-E_{\delta, \ell j}(\phi)\right)^{2}+\Gamma^{2}} .
$$

The factor 2 in Eqs.(8) and (9) comes from the spin degeneration of the system. The resonance of the differential conductance declines as the photon number $\mathrm{m}$ increases by the form $J_{m}^{2}\left(\Lambda_{L}\right)$. The resonance is strongly associated with the energy structure of the TCN and the applied photon energy. The resonant position is determined by $e V=E_{\delta, \ell j}(\phi)-m \hbar \omega_{L}$.

In the follows we perform the numerical calculations of differential conductance $d I / d V$ and tunneling current I versus source-drain bias $e V$, magnetic flux $\phi$, and the magnitude of ac field $\Lambda_{\gamma}$. The symmetric system is studied by setting $\Gamma_{L}=\Gamma_{R}=0.001 \gamma_{0}$. The photon energies of the fields are taken as $\hbar \omega_{L}=\hbar \omega_{R}=\hbar \omega=0.01 \gamma_{0}$, which is related to the frequency in the microwave region as $f=7.36 \times 10^{12} \mathrm{~Hz}$. We take the quantities $G_{0}=2 e^{2} / h$ and $I_{0}=2 e \gamma_{0} / h$ as the measurement scales of differential conductance and tunneling current. Figure 1 displays the structures of differential conductance for different TCN systems in the presence of external ac fields. The resonant structures of the differential conductance are modified due to the applied ac fields. The absorption and emission of photons induce novel resonant levels, and some levels of TCN are overlapped by the sideband. Therefore, we observe that some of the resonant peaks are suppressed by the ac fields. Diagrams (a), (b), and (c) are associated with the type II, type I, and type III TCNs. There is no energy gap in type I TCN system, 
but there exists large energy gap in type III TCN system. In the absence of magnetic flux, the energy gap of type II TCN can be calculated by $E_{g}=2 \gamma_{0}|1-z(\xi)|$, where $z(\xi)=\cos (\xi)-3^{1 / 2} \sin (\xi)$, and $\xi=b /\left(3^{1 / 2} D_{t}\right)[22]$. The energy gap of the $(7,7 ;-160,160)$ TCN is calculated to be $E_{g} \approx 68.77 \mathrm{meV}$. The photon energy applied to the system is $\hbar \omega=30.33 \mathrm{meV}$, which is much smaller than the energy gap, i.e., $\hbar \omega<<E_{g}$. Therefore, the electrons in the valence band can not jump to the conductance band for this system by absorbing photons. However, the applied ac fields provide new channels for electron to tunnel. This means that the electrons in the electrodes can transport through the system by applying the ac fields when $e V=0$. This procedure can be explained as the photon-electron pumping effect. The energy gap for the type III $(7,0 ;-160,320)$ TCN is about $E_{g} \approx 1.1 \mathrm{eV}$.

We present the differential conductance versus magnetic flux in Fig.2 to show the oscillation structure of $\mathrm{dI} / \mathrm{dV}$. The conductance is associated with the magnitude of external field as $\Lambda_{L}=0.8$ and frequency $f=0.01 \gamma_{0} / h$. The periodic oscillations can be seen for different TCNs with period $\phi_{0}$, and the oscillation structure is different for different types of TCN. In diagram (a), the solid and dotted curves are related to the zero-biased conductance of the type I $(7,7 ;-159,159)$ and type II $(7,7 ;-160,160)$ TCNs, respectively. For the type I TCN, a large resonant peak is accompanied with two small side resonant peaks in a period. These small side- peaks are arisen from the photon-assisted tunneling procedure. We can also note that the resonant structure is composed of the wave oscillation where two peaks form a bouble oscillation, and a resonant peak is embedded between the pair-peaks in a period. The small peaks located at $\phi=n \phi_{0}$ are caused by the photon emission and absorption. As the source-drain bias $e V=0.5 \gamma_{0}$, the oscillations of differential conductance are displayed in diagram (b). The oscillation structures are quite different from the ones related to zero-biased conductance. The oscillations shift and the side-peaks rise due to applying the voltage. However, the period is still $\phi_{0}$ for each of the oscillation. The differential conductance of type III TCN at $e V=0.5 \gamma_{0}$ is shown in diagram (c). One observes that the main peaks are split, and small peaks are embedded at the valleys due to applying external field.

The current oscillation structures versus the magnetic flux $\phi$ are shown in Fig.3 for different types of TCNs. We compare the current oscillation for the cases as the applied fields $\Lambda_{L}=\Lambda_{R}=0.8$ with the cases in the absence of the external fields $\Lambda_{L}=\Lambda_{R}=0$. Diagram (a) displays the current tunneling through the type II TCN. One observes that the magnitude of oscillation is suppressed, and the valleys are raised due to applying microwave field. Diagram (b) shows the current oscillation through the type I TCN system. Compared with the type II TCN system, we notice that the main peaks are split, and their magnitudes are smaller. The two current 
oscillations possess the phase shift by $\phi_{0} / 2$. The similar magnitude suppression and valley rising caused by the microwave field are also observed evidently. However, the oscillating structures for the type III TCN shown in diagram (c) are quite different from those in the type I and II TCN systems. In the absence of microwave field, the inverse resonance appears compared with diagrams (a) and (b). The current decreases abruptly around $\phi=n \phi_{0}$, and it changes mildly away from these points to form plateaus. The microwave field suppresses the valleys and splits the plateaus to form small valleys. The suppression of tunneling current is caused by the incoherent scatterings of photons with electrons.

Figure 4 shows the current-voltage characteristics of the type II and III TCN systems in the large region of source-drain bias $-\gamma_{0}<e V<\gamma_{0}$. The nonliear and obvious changes are observed. For the type II TCN system, the energy gap $E_{g}$ can not be observed obviously, since it is much smaller than the region of source-drain bias (the dotted curve). However, we can see the effect of energy gap for the type III TCN clearly in the I-V curve (the solid curve), since the energy gap is comparable with the source-drain bias. We present the current changes with the magnitude of microwave field in Fig. 5. It is seen clearly that the current changes mildly in the region of magnitude $0<\Lambda<2.6$, and then it declines rapidly as $\Lambda>2.6$. This indicates that the current can be suppressed very much by applying strong microwave field. The tunneling current may possess different current magnitudes for different types of TCN system, but the difference of current magnitude is smeared as the magnitude of microwave field is large enough.

We depict current-voltage and differential conductance versus source-drain bias in the small region $-5 \times$ $10^{-2} \gamma_{0}<\mathrm{eV}<5 \times 10^{-2} \gamma_{0}$ in Fig. 6 to display the photon-assisted tunneling behaviors obviously. The stairlike tunneling current is exhibited clearly in diagram (a). In the absence of microwave field, the steps are induced by the nature of TCN due to the discrete energy levels corresponding to the dotted curve. As the microwave fields are applied, novel steps are induced at $e V \approx 0, \pm 3.5 \times 10^{-2} \gamma_{0}$. This procedure is related to the absorption and emission of photons during the electron transport. The corresponding differential conductance is presented in diagram (b). We observe that in the absence of microwave field, two resonant peaks are located closely (pair-peak), and there exist large spaces between the pair-peaks. The magnitude of the differential conductance is about $2 G_{0}$ in this region. As the microwave field is applied, the magnitude of the conductance reduces to $1.6 G_{0}$, and the novel small peaks emerge between the pair-peaks. The steps in the I-V curves are associated with the resonant peaks of the differential conductance.

In summary, we have investigated the mesoscopic transport through the TCN system applied with ac fields to the electrodes. The photon-assisted tunneling current 
formulas are presented for evaluating the resonance and oscillation behaviors. The differential conductances are modified by the applied microwave fields. The conductance resonance takes place due to the electrons resonating in the quantum levels of TCN and side-band caused by the external ac fields. By changing the frequencies of the fields, we can obtain different photon energies, and hence the novel resonant conductance structure. The photon-assisted transport can be observed both in conductance oscillation and current oscillation with respect to the magnetic flux. The side peaks and current suppressions are the main effect of photon absorption and emission in the transport procedure. The stair-like currentvoltage characteristics are resulted from the quantum nature of TCN and applied microwave fields. The photonelectron pumping effect can be obtained by applying the microwave fields to the leads. The current suppression becomes significant as the magnitudes of external ac fields are large enough as $\Lambda>2.6$.

\section{ACKNOWLEDGMENTS}

This work was supported by a RGC grant from the SAR Government of Hong Kong under Grant No. HKU 7091/01P, and by the National Natural Science Foundation of China under the Grant No. 10375007.

[1] S. Iijima, Nature (London) 354, (1991) 56 .
[2] R. Saito, M. Fujita, G. Dresselhaus, and M.S. Dresselhaus, Appl. Phys. Lett. 60(1992) 2204 .

[3] R. Saito, G. Dresselhaus, and M.S. Dresselhaus, Physical properties of carbon nanotubes (Imperial College Press, London,1998).

[4] S. Roche, G. Dresselhaus, and M.S. Dresselhaus, and R. Saito, Phys. Rev. B 62(2000) 16092.

[5] J.P. Lu, Phys. Rev. Lett. 74(1995) 1123.

[6] H.K. Zhao, Eur. Phys. J. B 33(2003) 365.

[7] R. Martel, et al., Appl. Phys. Lett. 73(1998) 2447.

[8] S.J. Tans, A.R.M. Verschueren, and C. Dekker, Nature (London) 393(1998) 49 .

[9] Z. Yao, H.W.C. Postma, L. Balents, and C. Dekker, Nature (London) 402(1999) 273.

[10] J.W. Mintmire, B.I. Dunlap, and C.T. White, Phys. Rev. Lett. 68(1992) 631.

[11] S.J. Tans, M.H. Devoret, H. Dai, A. Thess, R.E. Smalley, L.J. Geerligs, and C. Dekker, Nature (London) 386(1997) 474 .

[12] C. Zhou, J. Kong, and H. Dai, Phys. Rev. Lett. $\mathbf{8 4}(2000) 5604$.

[13] T. W. Odom, J.L. Huang, P. Kim, and C.M. Lieber, Nature (London) 391(1998) 62.

[14] A. Bachtold, C. Strunk, J.P. Salvetat, J.M. Bonard, 
L. Forró, T. Nussbaumer and C. Schönenberger, Nature (London) 397(1999) 673.

[15] Y. Xue, and S. Datta, Phys. Rev. Lett. 83, 4844 (1999).

[16] H. Mehrez, J. Taylor, H. Guo, J. Wang, and C. Roland, Phys. Rev. Lett. 84(2000) 2682 ; C. Roland, M. B. Nardelli, J. Wang, and H. Guo, Phys. Rev. Lett. 84(2000) 2921.

[17] B.I. Dunlap, Phys. Rev. B 46(1992) 1933.

[18] S. Itoh, S. Ihara, and J. Kitakami, Phys. Rev. B 47, 1703 (1993); ibid. 47(1993) 12908.

[19] R.C. Haddon, Nature 388(1997) 31.

[20] R. Martel, H.R. Shea, and Ph. Avouris, Nature (London) 398(1999) 299.

[21] M.F. Lin, and D.S. Chuu, Phys. Rev. B 57(1998) 6731.

[22] H.K. Zhao, Phys. Lett. A 310(2003) 207; H.K. Zhao, Phys. Lett. A 308(2003) 226; H.K. Zhao, Phys. Lett. A $\mathbf{3 1 7}(2003) 329$.

[23] H.K. Zhao and G.v. Gehlen, Phys. Rev. B 58(1998) 13660; H.K. Zhao, and J. Wang, Phys. Rev. B 64(2001) 094505 .

[24] Q.F. Sun, J. Wang, and T.H. Lin, Phys. Rev. B 59(1999) 3831;59(1999) 13126.
[25] A.P. Jauho, N.S. Wingreen, and Y. Meir, Phys. Rev. B 50(1994) 5528.

\section{Figure Captions}

Fig. 1, The differential conductance versus the sourcedrain bias $e V$. The parameters are chosen as $\Lambda_{L}=$ $0.8, \hbar \omega=0.01 \gamma_{0}$, and $\phi=0$. Diagrams (a),(b) and (c) correspond to the $(7,7 ;-160,160),(7,7 ;-159,159)$ and (7,0;-160,320) TCNs, respectively.

Fig. 2, The differential conductance versus the magnetic flux $\phi$. The parameters are chosen as $\Lambda_{L}=$ $0.8, \hbar \omega=0.01 \gamma_{0}$. Diagram (a) represents the zero-biased conductance as $e V=0$ for the $(7,7 ;-160,160)$ (dotted curve) and (7,7;-159,159) (solid curve) TCNs, respectively. Diagram (b) represents the differential conductance as $e V=0.5 \gamma_{0}$ for the $(7,7 ;-160,160)$ (dotted curve) and $(7,7 ;-159,159)$ (solid curve) TCNs, respectively. Diagram (c) is the differential conductance as $\mathrm{eV}=0.5 \gamma_{0}$ for the $(7,0 ;-160,320) \mathrm{TCN}$.

Fig. 3, The tunneling current versus magnetic flux $\phi$ at $e V=0.5 \gamma_{0}$. The solid curves are associated with the cases as $\Lambda_{L}=\Lambda_{R}=0.8$, and the dotted curves are associated with the cases as $\Lambda_{L}=\Lambda_{R}=0$. Diagrams (a), (b), and (c) correspond to the $(7,7 ;-160,160),(7,7 ;-$ 159,159), and (7,0;-160,320) TCNs, respectively.

Fig. 4, The current-voltage characteristics of the system as $\Lambda_{L}=\Lambda_{R}=0.8$, and $\phi=0$. The solid and dotted curves are associated with $(7,0 ;-160,320)$ and $(7,7 ;-$ 160,160) TCNs, respectively. 
Fig. 5, The tunneling current versus the magnitude of ac fields for the case $\Lambda_{L}=\Lambda_{R}=\Lambda$ as $e V=0.8 \gamma_{0}$ and $\phi=0$. The solid and dotted curves are related to the (7,7;-160,160) and (7,0;-160,320) TCNs, respectively.

Fig. 6, The current-voltage characteristics and corresponding differential conductance of $(7,7 ;-160,160)$ TCN versus source-drain bias $\mathrm{eV}$. The dotted and solid curves are related to the parameters $\Lambda_{L}=\Lambda_{R}=0$, and $\Lambda_{L}=\Lambda_{R}=0.8$, respectively. 